\title{
Variables que determinan el valor de las exportaciones de un recurso natural no renovable: caso del cobre peruano periodo 2001-2014
}

Variables that determine the value of the exports of a non-renewable natural resource: case of peruvian copper. period 2001-2014

\author{
Oscar Fernando Navarro Angeles ${ }^{1}$
}

\section{Resumen}

Debido a la significativa contribución del cobre en el valor total de las exportaciones del Perú, es necesario identificar las variables que la influyen para el período 2001-2014, se ha determinado su comportamiento y relación con variables relevantes. Para ello, se ha utilizado el coeficiente de correlación entre las series, la regresión lineal múltiple, y la Prueba de Grange. La demanda de cobre por parte de China, Japón, Alemania y Corea del Sur, ha sido importante en el comportamiento fluctuante en el corto plazo y creciente en el largo plazo del valor de las exportaciones del cobre peruano.

Palabras clave: variables; Exportaciones; Importaciones; Recurso; Cobre; Perú; Precio; Demanda Mundial; Mercado; Correlación; Fluctuación.

\begin{abstract}
Due to the significant contribution of copper to the total value of Peruvian exports, it is necessary to identify the variables that influence it for the period 2001-2014, its behavior and relation with relevant variables have been determined. For this purpose, the correlation coefficient between the series, the multiple linear regression, and the Grange test were used. The demand for copper by China, Japan, Germany and South Korea has been important in the fluctuating behavior in the short term and increasing in the long term the value of exports of Peruvian copper.
\end{abstract}

Keywords: variables; Exports; Imports; Resource; Copper; Peru; Price; Worldwide Demand; Market; Correlation; Fluctuation.

\section{Introduccion}

En el período 2001-2014, la participación del valor de las exportaciones de cobre, en el total de las exportaciones mineras del Perú, fue una de las más significativas, pasando de 30,8\% (2001) a 43,2\% (2014) (Figura 1) y en relación al

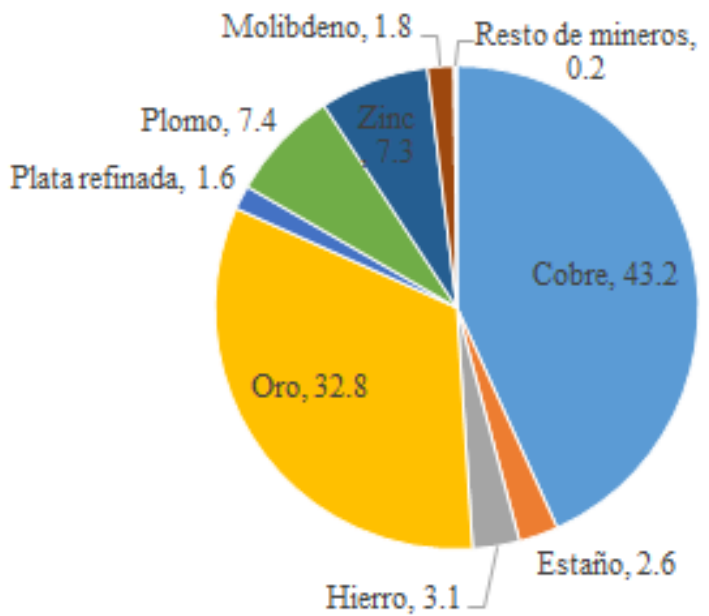

Figura 1. Participación porcentual del valor de las exportaciones mineras del Perú, año 2014 valor total de las exportaciones de productos tradicionales (pesqueros, agrícolas, mineros, petróleo y gas natural), la participación del valor de las exportaciones de cobre fue de $32,1 \%$ en el 2014.

En términos monetarios, según el Banco Central de Reserva del Perú (2006), el valor Libre a Bordo (Free on board) de las exportaciones de cobre del Perú aumentó de 986 millones de US\$ (2001) a 8.875 millones de US\$ (2014), implicando un crecimiento de $800 \%$ en un período de 13 años, evidenciando una tendencia creciente a largo plazo (Figura 2).

Pero el valor de las exportaciones de cobre ha mostrado un comportamiento fluctuante en el corto plazo, observable en su tasa de crecimiento anual (Figura 3), la cual ha oscilado entre 96,8\% (2004) y -18,4\% (2009). Estas fluctuaciones se explicarían por el comportamiento del precio y el volumen de las exportaciones de cobre, por lo que es importante identificar los factores que han influenciado en las referidas variables durante el período del estudio.

Debido a la significativa importancia de la contribución de las exportaciones de cobre en la disponibilidad de divisas para el país, que según el Banco Central de Reserva 
del Perú (2006) representó el 22.5\% del valor total de las exportaciones (tradicionales y no tradicionales) en el 2014, y dado el comportamiento fluctuante en el corto plazo y la magnitud creciente en el largo plazo del valor de las

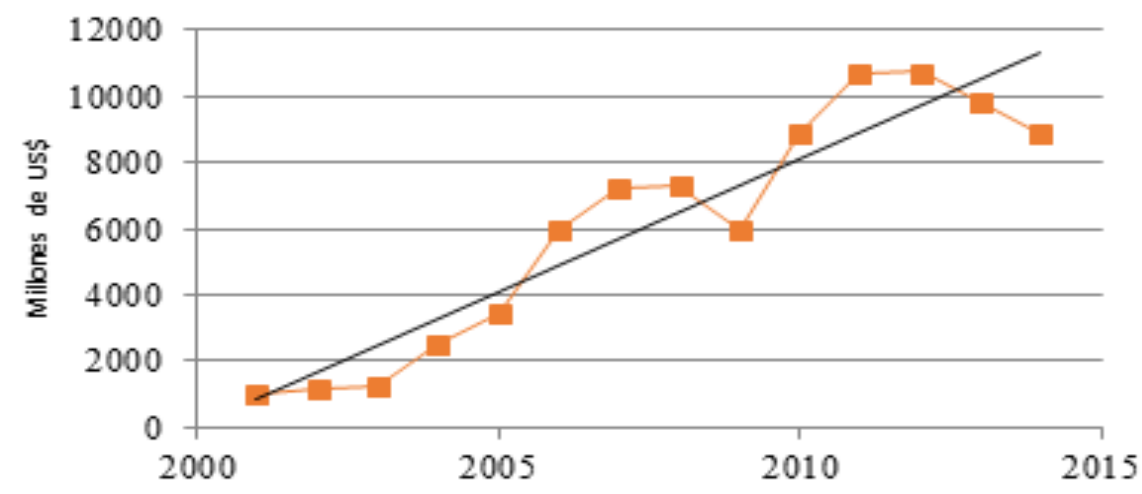

Figura 2. Valor FOB de las exportaciones de cobre del Perú, período 2001-2014 los principales países de destino, la demanda mundial de cobre y el Producto Bruto Interno (PBI) mundial.

Método Inductivo: en base al análisis de los datos históricos se buscó determinar el comportamiento de las variables, y las relaciones de correlación y causalidad entre las variables.

La metodología empleada comprendió los siguientes pasos:

- Para el estudio del comportamiento de las variables relevantes se utilizó series trimestrales y anuales del período 2001-2014 provenientes de fuentes secundarias (Banco Central de Reserva del Perú (2006), Banco Mundial (s.f.), The Observatory of Economic Complexity (2014)). Asimismo, se analizó la evolución

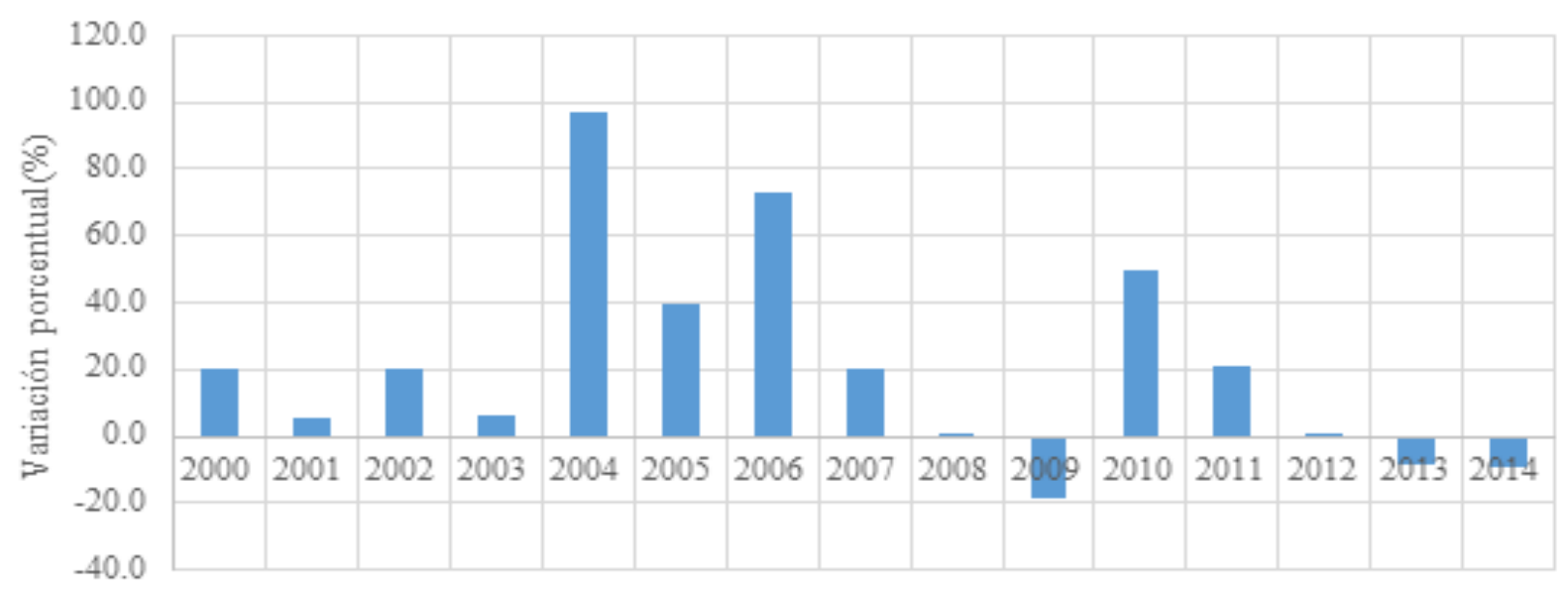

Figura 3. Variación porcentual del valor de las exportaciones de cobre del Perú, período 2000-2014

exportaciones de cobre del Perú para el período 20012014, El objetivo del presente estudio fue determinar la relación del valor de las exportaciones de cobre del Perú con las variables de influencia como el precio internacional del cobre y la demanda de cobre peruano por parte de los principales países de destino (China, Japón, Alemania y Corea del Sur).

\section{Materiales y métodos}

Siendo el objetivo del presente estudio identificar las variables que han determinado el valor de las exportaciones de cobre del Perú en el período 2001-2014, los métodos de investigación empleados han sido el Deductivo e Inductivo.

Método Deductivo: sobre la base de la teoría económica, se buscó explicar las causas del comportamiento del valor de las exportaciones de cobre, para lo cual se identificó como variables relevantes e influyentes (explicativas): el precio internacional del cobre, el volumen de las exportaciones de cobre del Perú, la demanda del cobre peruano por parte de de las series de tiempo, lo que permitió determinar el comportamiento fluctuante a corto plazo, las tendencias a largo plazo, y las relaciones entre variables.

- Para los estudios de correlación, que buscan establecer relaciones directas e inversas entre variables, se determinó el coeficiente de correlación entre las series trimestrales y anuales, y se realizó algunas regresiones lineales múltiples.

- Para los estudios de causalidad entre variables, se aplicó la Prueba de Granger entre algunas series trimestrales. La metodología empleada permitió identificar las relaciones existentes entre las variables. Para ello, se utilizó el Quantitative Micro Software EVIEWS.

\section{Resultados y discusión}

Evolución del valor de las exportaciones de cobre del Perú

En el corto plazo, el valor trimestral de las exportaciones de cobre del Perú, durante el período 2001-2014, mostró 
un comportamiento estacional, observándose tasas de crecimiento negativo en el primer trimestre de 11 de los 14 años que comprende el período de estudio (Figura 4). Este comportamiento estacional, con tasas de crecimiento negativo, coincide con los meses de invierno duro de China, quien es el mayor importador del cobre peruano, cuya demanda ha ido creciendo durante el período de estudio, y en el 2014 demandó el 49\% del valor total de las exportaciones del cobre peruano. Asimismo, el referido comportamiento estacional coincide con los meses fríos de invierno de Japón, Alemania y Corea del Sur, países que conjuntamente demandaron el $23 \%$ del valor total de las exportaciones del cobre peruano en el 2014.
El valor de las exportaciones es el resultado de la multiplicación del volumen de las exportaciones por el precio del cobre, su comportamiento estacional se explicaría principalmente por el comportamiento del volumen de las exportaciones, las que también mostraron tasas de crecimiento negativo en el primer trimestre de 11 de los 14 años que comprende el estudio (Figura 5), coincidiendo con el comportamiento del valor de las exportaciones del cobre peruano (Figura 4).

En el caso del precio del cobre, su tasa de crecimiento negativo en el primer trimestre coincidió con las del valor de las exportaciones solo en 4 de los 14 años que comprende el estudio (Figura 6), por tanto, el comportamiento estacional del valor de las exportaciones de cobre del Perú (tasa de

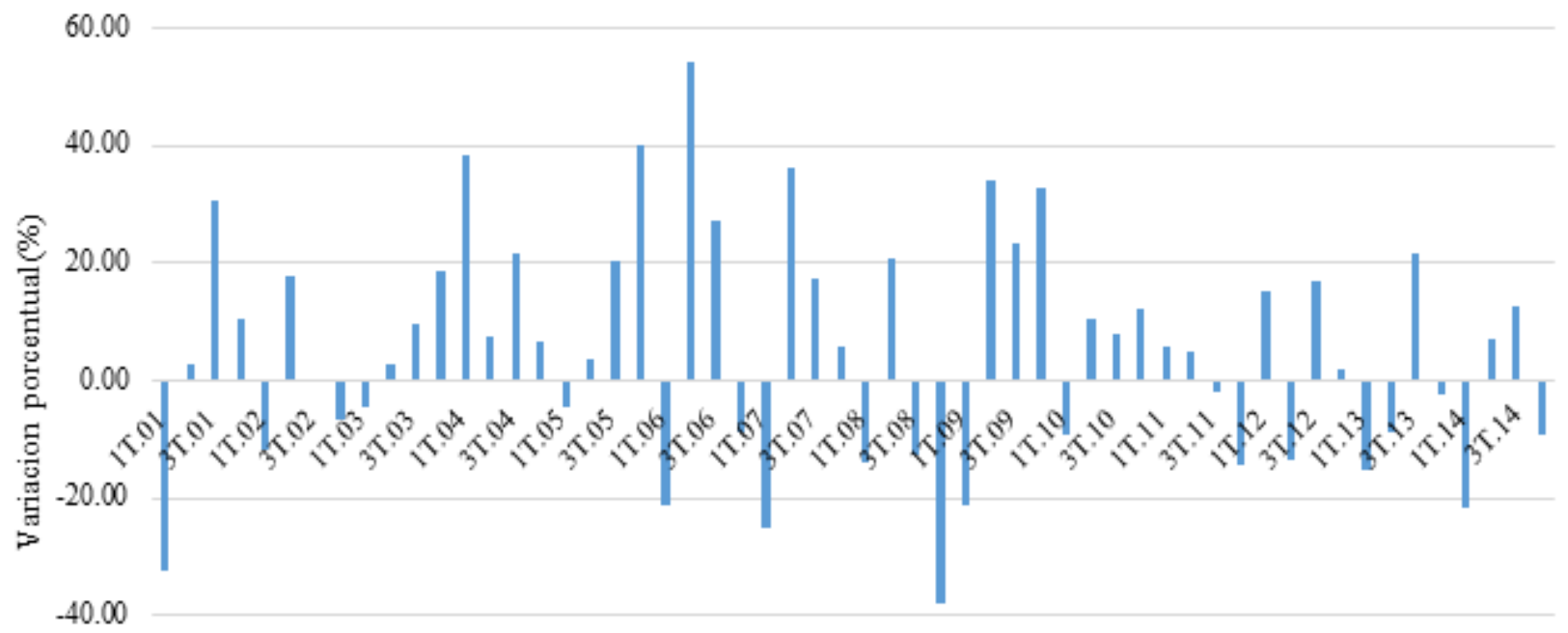

$-60.00$

\section{Trimestres}

Figura 4. Variación porcentual del valor trimestral de las exportaciones de cobre del Perú, período 2001-2014

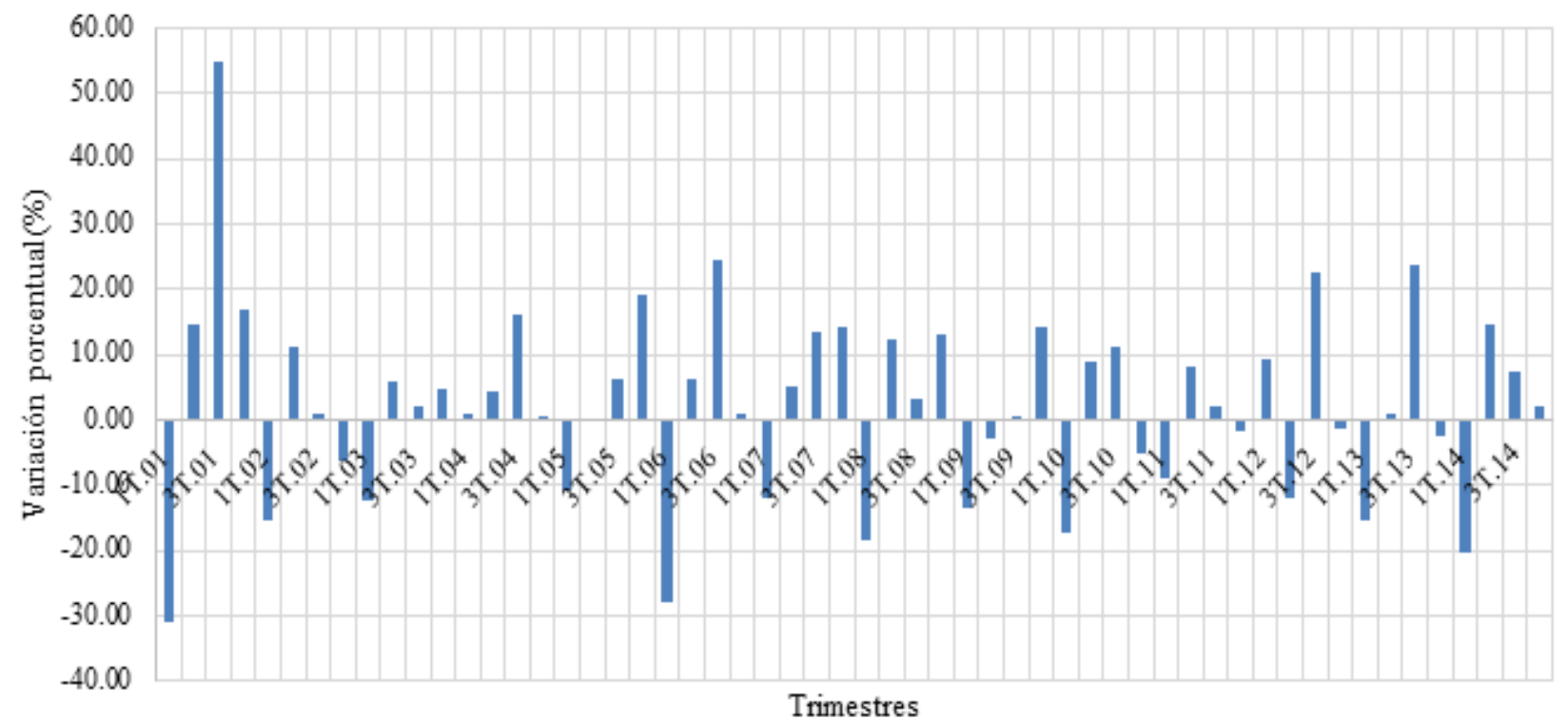

Figura 5. Variación porcentual del volumen trimestral de las exportaciones de cobre del Perú, período 2001-2014 
crecimiento negativo en los primeros trimestres de cada año) se explicaría principalmente por el comportamiento del volumen de las exportaciones de cobre.

En el largo plazo, el valor trimestral desestacionalizado de las exportaciones de cobre del Perú mostró una tendencia creciente en el período 2001-2011. Pasando de 241,74 millones US\$ (1er. trim-2001) a 2.051,91 millones US\$ (1 er. trim-2008), luego tuvo una caída significativa, llegando a 1.401,6 millones de US\$ (1er. trim-2009), coincidiendo con la crisis económica mundial que inició el 2008 y continuó el 2009. Luego se recuperó llegando a 2.693,9 millones de US\$ (3er trim-2011), y finalmente mostró una tendencia decreciente hasta el 2014 (Figura7).

Pero es notorio el incremento significativo de nivel del valor trimestral desestacionalizado de las exportaciones de cobre del Perú en el quinquenio 2010-2014, evidenciado en su promedio trimestral (Tabla 1).

Evolución del volumen de las exportaciones de cobre del Perú

En el largo plazo, el volumen anual de las exportaciones de cobre del Perú (período 2001-2014) mostró una tendencia creciente, pasando de 685,8 mil tm (2001) a 1.402,4 mil tm (2014), evidenciando un incremento del 104,8\%. Además, el volumen de las exportaciones de cobre experimentó un incremento de nivel, hasta el 2006 el volumen anual estaba por debajo de las $1.000 \mathrm{mil} \mathrm{tm}$, hasta el 2011 por debajo de las 1.270 mil tm, y a partir del 2012 por encima de las 1.400 mil tm (Figura 8). Este sucesivo incremento del nivel del volumen de las exportaciones de cobre evidenció el fortalecimiento de la capacidad productiva de las empresas mineras que operan en el país.

Tabla 1. Promedio trimestral del valor desestacionalizado por quinquenios de las exportaciones de cobre del Perú, período 2001-2014

\begin{tabular}{cc}
\hline Quinquenio & Promedio trimestral (millones de US\$) \\
\hline $2001-2004$ & 320,0 \\
$2005-2009$ & 1.496 .7 \\
$2010-2014$ & $2.435,4$ \\
\hline
\end{tabular}

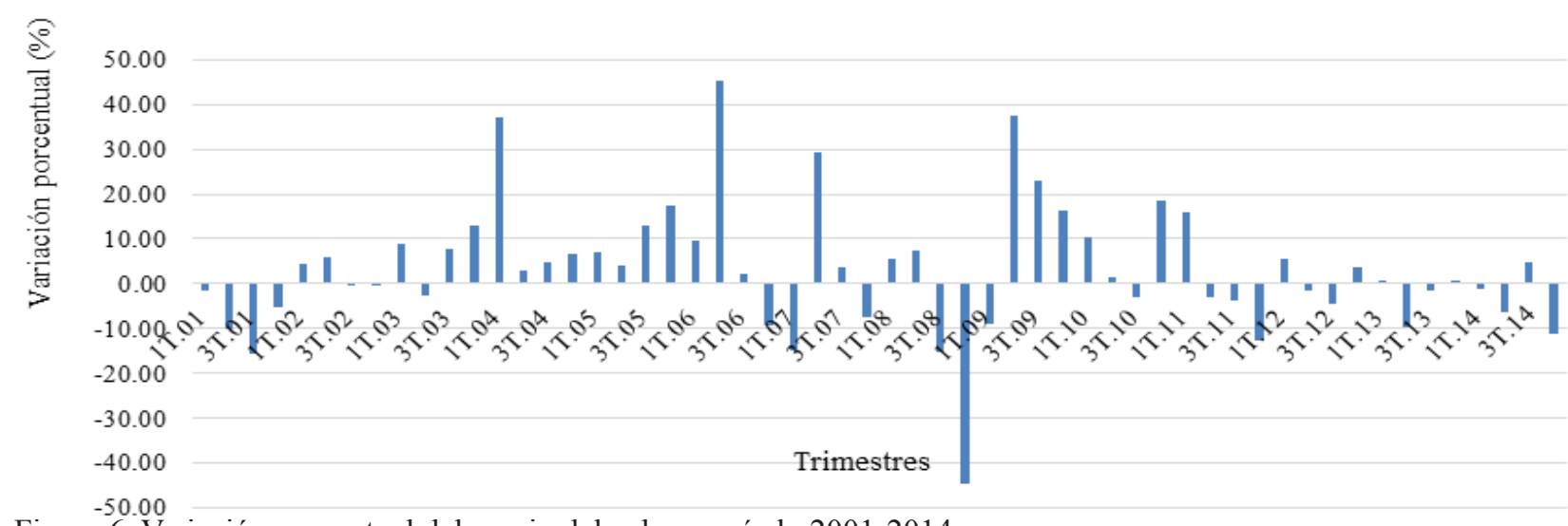

Figura 6. Variación porcentual del precio del cobre, período 2001-2014

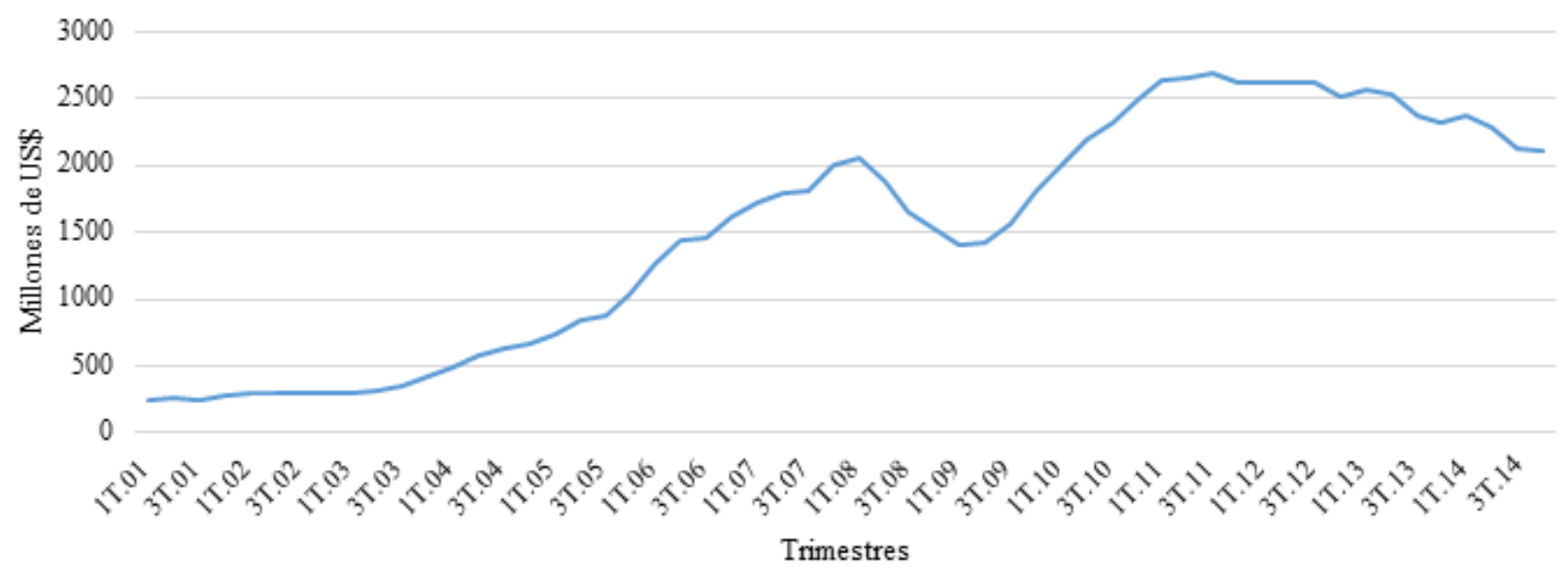

Figura 7. Valor trimestral desestacionalizado de las exportaciones de cobre de Perú, período 2001-2014 
Cabe indicar que la tendencia creciente del volumen anual de las exportaciones de cobre observada en los años 2007 y 2008, se detuvo en los años 2009, 2010 y 2011, coincidiendo con la crisis económica mundial, y volvió a crecer en el año 2012, para luego detenerse en los años 2013 y 2014 (Tabla 2).

Como ya se mencionó, en el corto plazo, el comportamiento del volumen de las exportaciones de cobre del Perú ha sido fluctuante, mostrando crecimiento negativo prácticamente en el primer trimestre de 11 de los 14 años del período de estudio, y un mayor crecimiento positivo en el tercer trimestre de 7 de los 14 años, evidenciando un comportamiento estacional (Figura 5).

Tabla 2. Variación porcentual del valor y volumen de las exportaciones de cobre del Perú, y del precio del cobre, período 2000-2014

\begin{tabular}{cccc}
\hline & $\begin{array}{c}\text { Variación } \\
\text { porcentual (\%) } \\
\text { del valor de las } \\
\text { exportaciones }\end{array}$ & $\begin{array}{c}\text { Variación porcentual } \\
(\%) \text { del volumen de } \\
\text { las exportaciones }\end{array}$ & $\begin{array}{c}\text { Variación } \\
\text { porcentual }(\%) \\
\text { del precio }\end{array}$ \\
\hline 2000 & 20.1 & 1.5 & 18.3 \\
2001 & 5.7 & 29.6 & -18.5 \\
2002 & 20.4 & 25.2 & -3.8 \\
2003 & 6.2 & -8.3 & 15.8 \\
2004 & 96.8 & 19.5 & 64.7 \\
2005 & 40.0 & 4.6 & 33.8 \\
2006 & 72.7 & -0.4 & 73.3 \\
2007 & 20.4 & 14.4 & 5.2 \\
2008 & 0.8 & 10.8 & -9.0 \\
2009 & -18.4 & 0.2 & -18.6 \\
2010 & 49.6 & 0.8 & 48.4 \\
2011 & 20.7 & 0.5 & 20.2 \\
2012 & 0.1 & 11.4 & -10.1 \\
2013 & -8.5 & -0.1 & -8.4 \\
2014 & -9.6 & -0.1 & -9.5 \\
\hline
\end{tabular}

\section{Evolución del precio del cobre exportado por Perú}

El precio FOB del cobre ha mostrado una tendencia creciente del 4to.trim-2001 al 2do.trim-2008, pasando de 57,65 ctvs.US\$/libra a 334,45 ctvs.US\$/libra respectivamente, habiéndose incrementado en $480 \%$. Luego desciende a
142,01 ctvs.US\$/libra en el 1er.trim-2009 (coincidiendo con la crisis económica mundial), y asciende a 415,68 ctvs. US\$/libra en el 1er.trim-2011. Finalmente desciende en el resto del período, cayendo a 264,7 ctvs.US\$/libra en el 4 to. trim-2014 (Figura 9).

A pesar de las fluctuaciones que ha experimentado el precio del cobre, ha logrado consolidar niveles cada vez mayores, pues el precio promedio anual ha pasado de 157 ctvs.US\$/libra (2003-2006) a 273.5 ctvs.US\$/libra (20072010), y finalmente a 334 ctvs.US\$/libra (2011-2014), lo que ha sido un estímulo al incremento del nivel de las exportaciones de cobre.

\section{Relación entre el valor y el volumen de las exportaciones de cobre del Perú}

En el largo plazo, el volumen y el valor de las exportaciones de cobre del Perú mostró una relación directa, con excepción de algunos años. En el caso del volumen de las exportaciones, ha ido alcanzado y consolidando mayores niveles, los que se han mantenido a pesar de las caídas que el precio del cobre ha experimentado en diversos años (2008, 2009, 2013 y 2014), caídas que han afectado negativamente al valor de las exportaciones de cobre en los referidos años (Figura 10). Rojas (2017) resalta que con mayores volúmenes de producción y eficiencia en la estructura de costos, se puede enfrentar a la baja ley del mineral y la caída de los precios de los commodities.

Basándonos en las series trimestrales (Figura11), hay una relación directa entre el valor y el volumen de las exportaciones de cobre del Perú en el periodo 2001-2007 (coeficiente de correlación de 0.778), en el periodo 2008-2009 no existe relación (coeficiente de correlación de -0.095), y en el periodo 2010-2014 la relación directa se restablece (coeficiente de correlación de 0.414). Cabe indicar que en el periodo 2008-2009, el precio del cobre influenció significativamente en el valor de las exportaciones, evidenciado por el coeficiente de correlación de 0.956. Y como lo reporta el Banco Central de Reserva del Perú (2006), que las mayores ventas del cobre reportado en el 2006 es explicado por el incremento en el precio promedio del cobre.

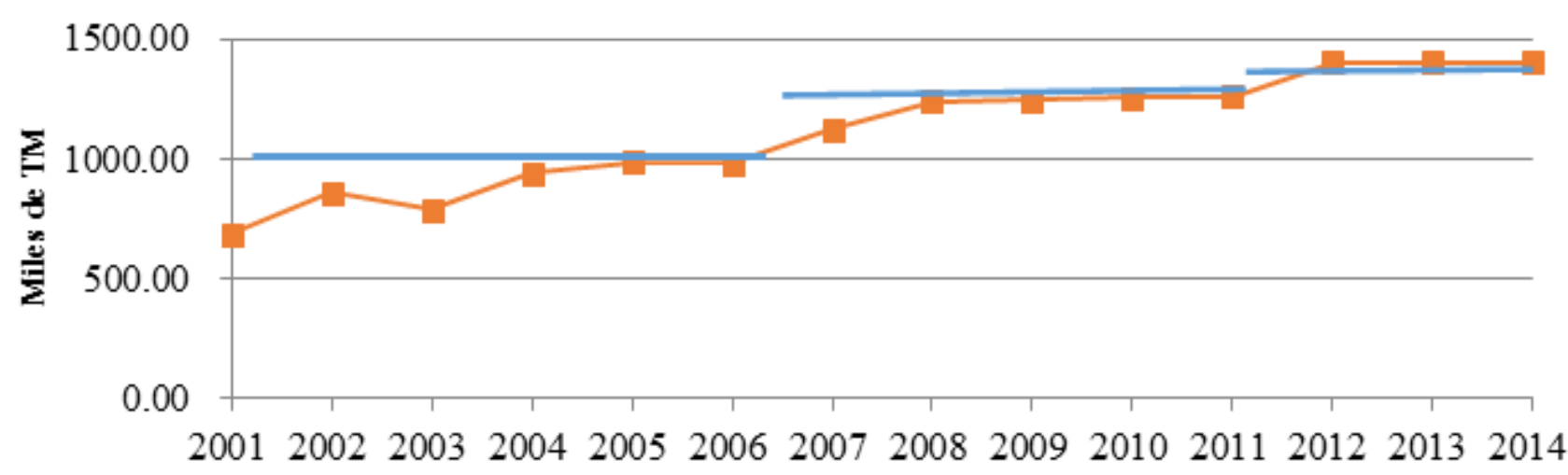

Figura 8. Volumen anual de las exportaciones de cobre del Perú, período 2001-2014 


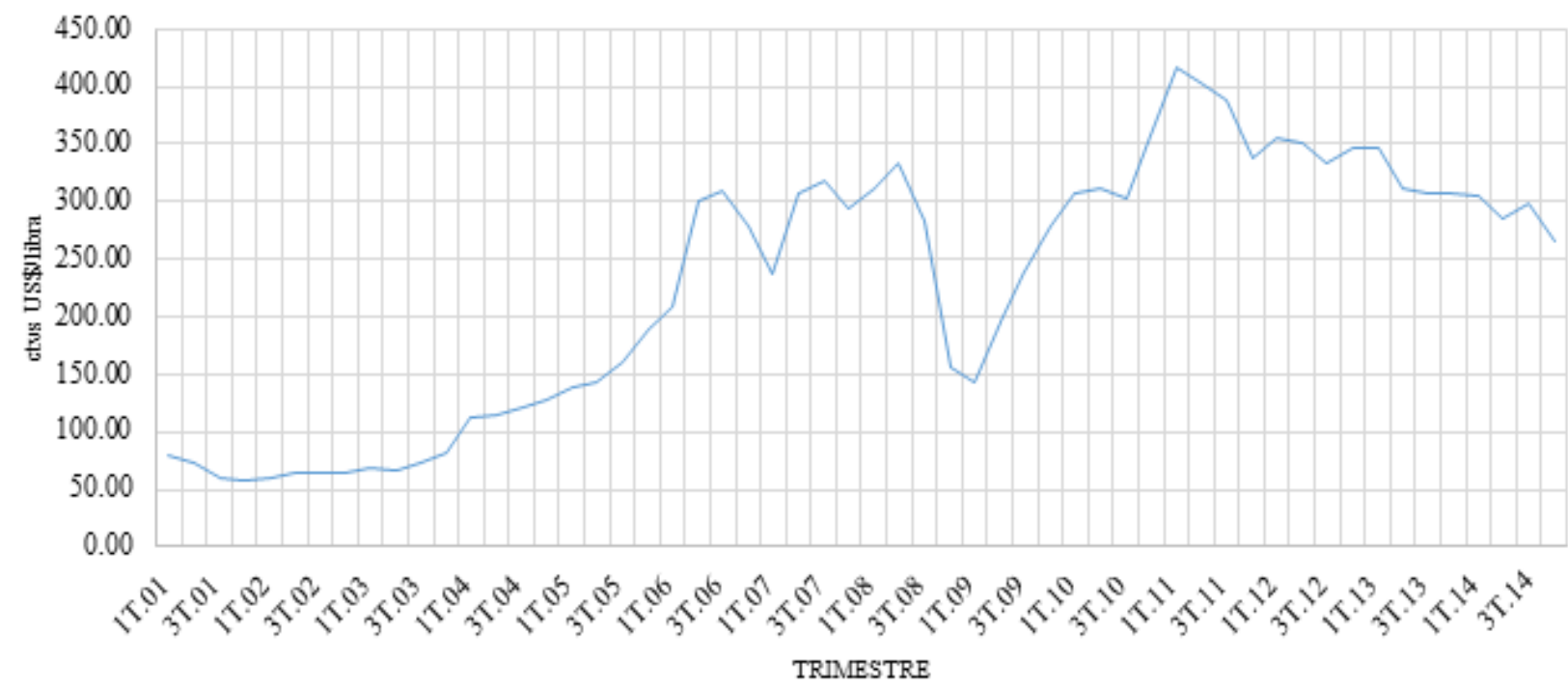

Figura 9. Evolución del precio fob del cobre, período 2001-2014

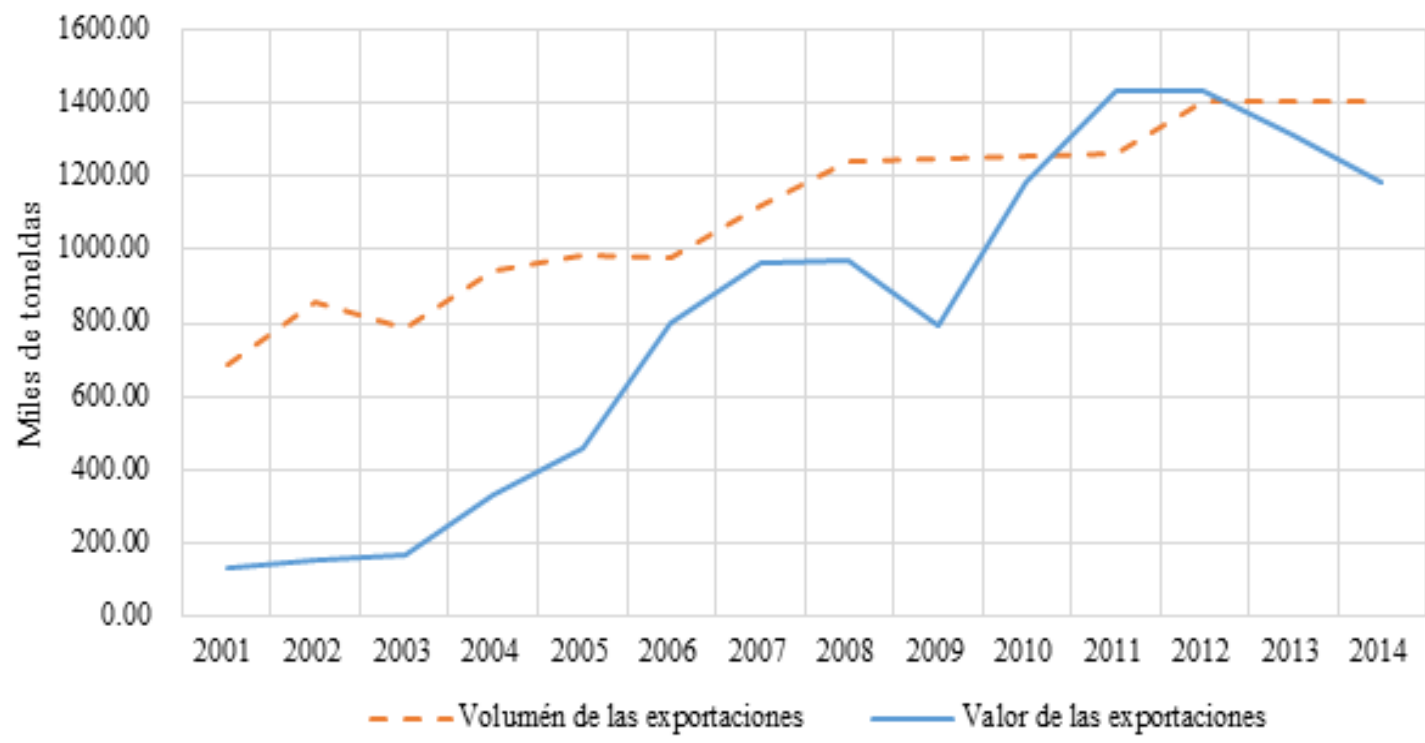

12000.00

10000.00

8000.00

6000.00

4000.00

2000.00

0.00

Figura 10. Volumen y valor anual de las exportaciones de cobre del Perú, período 2001-2014

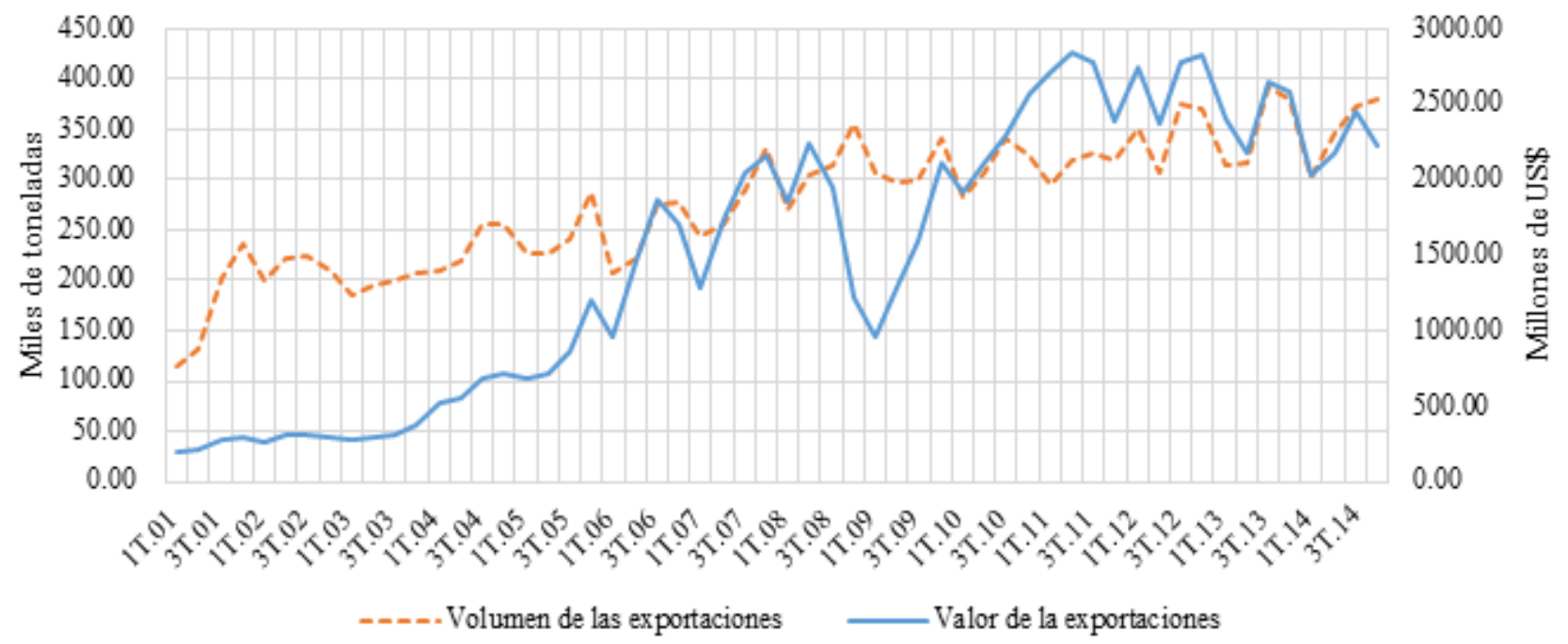

Figura 11. Volumen y valor de las exportaciones trimestrales de cobre del Perú, período 2001-2014 


\section{Relación entre el volumen de las exportaciones y el} precio del cobre

Se encuentra una relación directa entre las series trimestrales del volumen de las exportaciones y el precio del cobre para el período 2001-2008 (coeficiente de correlación 0.655). Esto evidenciaría que ante una mayor demanda internacional de cobre habría provocado un aumento del volumen de exportación y del precio, pues en el referido periodo el volumen anual de exportación de cobre del Perú pasó de 685,9 miles tm (2001) a 1.243,09 miles tm (2008). Pero el período 2009-2014, el coeficiente de correlación es 0.054 , evidenciando ninguna relación entre el volumen de las exportaciones y el precio del cobre, cabe indicar que en el referido período el volumen anual de las exportaciones prácticamente no creció (2009: 0.2\%; 2010: $0.8 \%$; 2011: $0.5 \% ; 2013:-0.1 \% ; 2014:-0.1 \%$ ), salvo el año 2012 (11.4\%). Rojas (2017) reporta que la empresa minera Cerro Verde, en el año 2009, dada la crisis en el mundo, paro el proceso de expansión.

Al considerar las series trimestrales desestacionalizadas del volumen de las exportaciones y el precio del cobre, cobre, indicaría que hay otros factores que determinaron el volumen de las exportaciones de cobre del Perú, lo que daría lugar a considerar el comportamiento de la demanda de cobre por parte de los principales países de destino del cobre peruano.

Al realizar la Prueba de Grange (Prueba de Causalidad), entre las series trimestrales del volumen de las exportaciones y del precio del cobre (considerando un trimestre de rezago), se determinó que el precio del cobre Causa-Grange al volumen de las exportaciones (Tabla 3). Y similar resultado se obtuvo cuando se realiza la Prueba Grange entre las series trimestrales desestacionalizadas del volumen de las exportaciones y del precio del cobre, considerando un trimestre de rezago, (Tabla 4).

\section{Valor de las exportaciones de cobre del Perú por mercados de destino}

En el periodo de estudio 2001-2014, China ha sido el principal destino de las exportaciones del mineral de cobre peruano, pasando de 26\% (2001) a 49\% (2014) del valor total de las exportaciones, y en valores monetarios

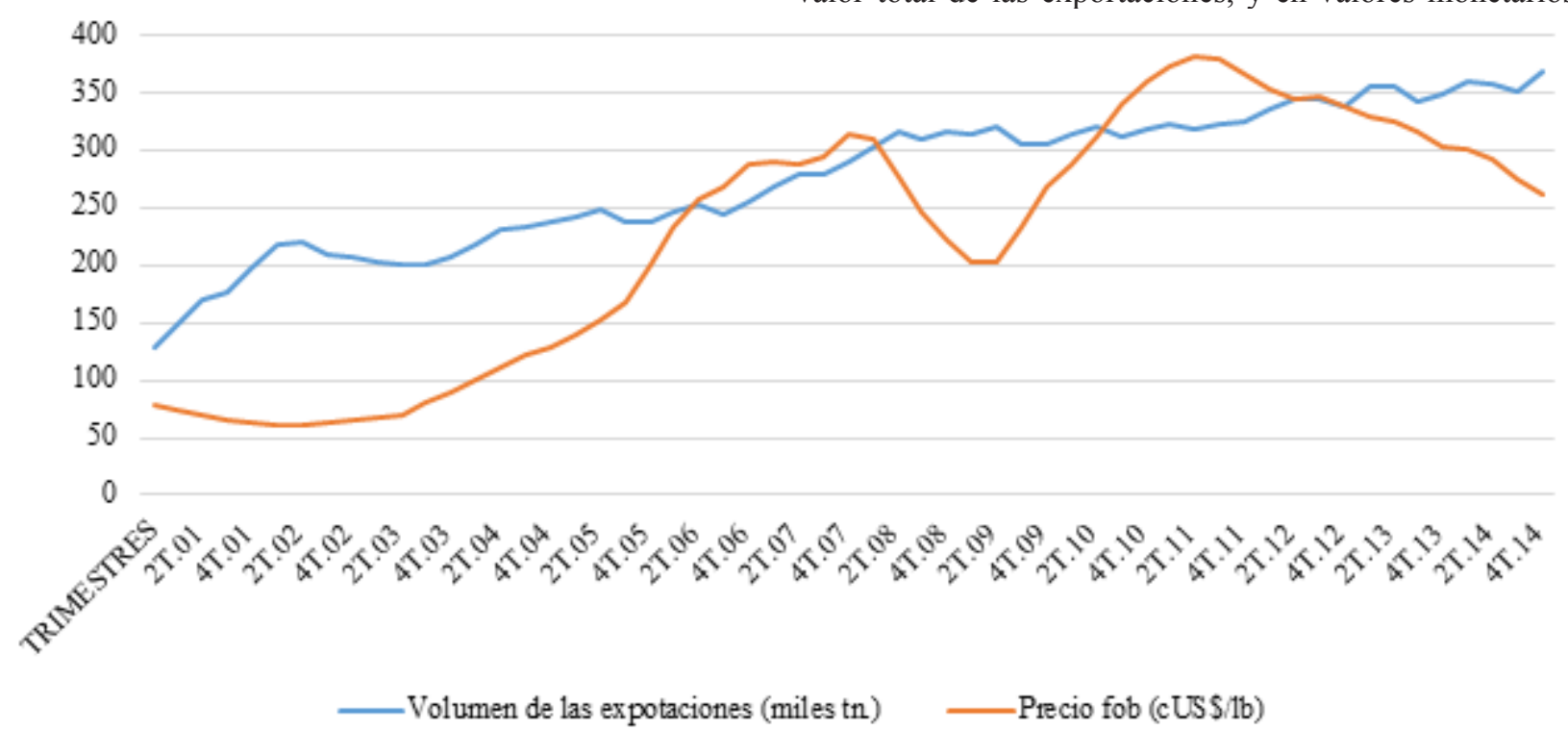

Figura 12. Series trimestrales desestacionalizadas del volumen de las exportaciones y el precio FOB del cobre, período 2001-2014

para el período 2001-2007, se observa que ambas series muestran una tendencia creciente (Figura 12). Pero el volumen de las exportaciones siguió creciendo aun cuando el precio cayó de 308,70 ctvs.US\$/libra (1 er.trim-2008) a 202.56 ctvs.US\$/libra (2do.trim2009), y también cuando el precio mostró una tendencia decreciente a partir del 2do.trim-2011 al 2014. Esta relación inversa en algunos períodos o falta de relación en otros, entre las tendencias del volumen de las exportaciones y del precio del
Tabla 3. Prueba de Grange entre el volumen de exportación y el precio del cobre (series puras)

Pairwise Granger Causality Tests

Data: 05/16/17 Time: 21:41

Sample: 2001Q1 2014Q4

Lags: 1

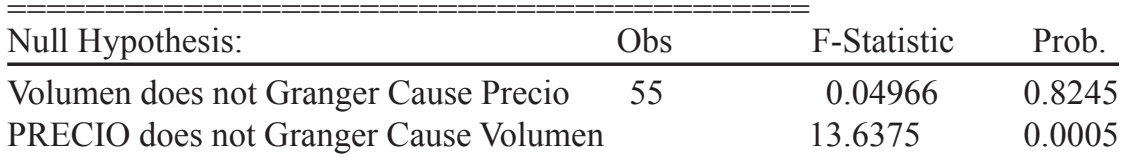


Tabla 4. Prueba de Grange entre el volumen de exportación y el precio del cobre (series desetacionalizas)

Pairwise Granger Causality Tests

Data: 05/16/17 Time: 21:52

Sample: 2001Q1 2014Q4

Lags: 1

\begin{tabular}{|c|c|c|c|}
\hline Null Hypothesis: & Obs & F-Statistic & Prob. \\
\hline Volumen does not Granger Cause Precio & 55 & 2.55025 & 0.1163 \\
\hline PRECIO does not Granger Cause Volumen & & 6.61989 & 0.0130 \\
\hline
\end{tabular}

se incrementaron de 57.6 millones US\$ (2001) a 3.410 millones US\$ (2014). El segundo destino en importancia ha sido Japón, que durante el período de estudio, en promedio anual representó el $18.1 \%$, seguido de Alemania con el $10.3 \%$ y Corea del Sur con el 6.1\%. En el 2001, estos cuatro países importaron el $53.5 \%$ del valor total de las exportaciones de mineral de cobre del Perú, lo que posteriormente aumentó a 72\% en el 2014 (Tabla 5).

El comportamiento del valor de las exportaciones de cobre del Perú hacia los cuatro principales países de destino (China, Japón, Alemania, Corea del Sur) durante el período 2001-2014, muestra una tendencia creciente en el tiempo, con excepción de los años 2008, 2009, 2013 y 2014, en los cuales el valor de las exportaciones no crecieron o disminuyeron (Figura 13). Cabe indicar que la
El valor de las importaciones totales de mineral de cobre (proveniente del mundo) por parte de los países de destino (China, Japón, Alemania y Corea del Sur) está relacionado con sus respectivos Producto Bruto Interno (PBI), pues para el período 2002-2014, el coeficiente de correlación entre la tasa de crecimiento anual del valor total de sus importaciones de cobre (provenientes del mundo) con la tasa de crecimiento de sus respectivos PBI, es significativa (Tabla 7). Esto debido a los diversos usos que se da al cobre en el sector eléctrico (generación y distribución de la energía eléctrica), en la construcción, en la industria manufacturera y en las telecomunicaciones (Moneda, 2016). Por lo que al incrementarse el nivel de actividad económica de cada país, se espera que la demanda de cobre aumente.

Tabla 6. Coeficiente de correlación entre las tasas de crecimiento anual del valor de las exportaciones de cobre del Perú hacia los principales países de destino, período 2002-2014

\begin{tabular}{cccc}
\hline Países & Japón & Alemania & Corea del Sur \\
\hline China & 0.88 & 0.73 & 0.47 \\
Japón & & 0.76 & 0.55 \\
Alemania & & & 0.42 \\
\hline
\end{tabular}

Tabla 5. Destino del valor de las exportaciones de cobre del Perú por países (millones US\$), período 2001-2014

\begin{tabular}{|c|c|c|c|c|c|c|c|c|c|c|}
\hline & China & $\%$ & Japón & $\%$ & Alemania & $\%$ & COrea del Sur & $\%$ & Otros países & Total exportado \\
\hline 2001 & 57.6 & 26 & 37.2 & 17 & 15.5 & 7 & 7.8 & 3.5 & 102.9 & 221 \\
\hline 2002 & 113 & 26 & 81.5 & 19 & 66.1 & 15 & 43.4 & 10 & 130 & 434 \\
\hline 2003 & 161 & 39 & 80.2 & 19 & 46.1 & 11 & 44.7 & 11 & 84 & 416 \\
\hline 2004 & 408 & 37 & 177 & 16 & 134 & 12 & 64.5 & 5.8 & 326.5 & 1110 \\
\hline 2005 & 503 & 35 & 207 & 15 & 130 & 9.2 & 71.1 & 5 & 508.9 & 1420 \\
\hline 2006 & 924 & 33 & 422 & 15 & 337 & 12 & 133 & 4.7 & 1024 & 2840 \\
\hline 2007 & 1560 & 34 & 1310 & 28 & 480 & 10 & 291 & 6.3 & 989 & 4,630 \\
\hline 2008 & 1560 & 32 & 1120 & 23 & 492 & 10 & 123 & 2.5 & 1595 & 4890 \\
\hline 2009 & 1380 & 35 & 883 & 22 & 414 & 10 & 300 & 7.6 & 993 & 3970 \\
\hline 2010 & 1700 & 27 & 1150 & 19 & 731 & 12 & 433 & 7 & 2186 & 6200 \\
\hline 2011 & 2420 & 31 & 1300 & 17 & 866 & 11 & 553 & 7.1 & 2601 & 7740 \\
\hline 2012 & 3500 & 42 & 1450 & 18 & 934 & 11 & 467 & 5.6 & 1929 & 8280 \\
\hline 2013 & 3390 & 44 & 1170 & 15 & 546 & 7 & 408 & 5.2 & 2276 & 7790 \\
\hline 2014 & 3410 & 49 & 754 & 11 & 503 & 7.3 & 328 & 4.7 & 1935 & 6930 \\
\hline Promedio & & 35 & & 18.1 & & 10.3 & & 6.1 & & \\
\hline
\end{tabular}

economía mundial disminuyó su tasa de crecimiento anual en los referidos años: $1.84 \%$ (2008), $-1.70 \%$ (2009), $2.47 \%$ (2013), y 2.69\% (2014).

El comportamiento del valor de las exportaciones de cobre del Perú hacia cada uno de los cuatro principales países de destino mostró relación directa entre ellos, evidenciándose en altos coeficientes de correlación entre sus tasas de crecimiento anual, especialmente entre China, Japón y Alemania, y en menor medida con Corea del Sur (Tabla 6).
Tabla 7. Coeficiente de correlación entre la tasa de crecimiento anual del total de las importaciones de mineral de cobre de cada país y su respectivo PBI, período 20022014

\begin{tabular}{ccccc}
\hline Período & China & Japón & Alemania & Corea del Sur \\
\hline 2002-2014 & 0.434 & 0.566 & 0.446 & 0.313 \\
\hline
\end{tabular}




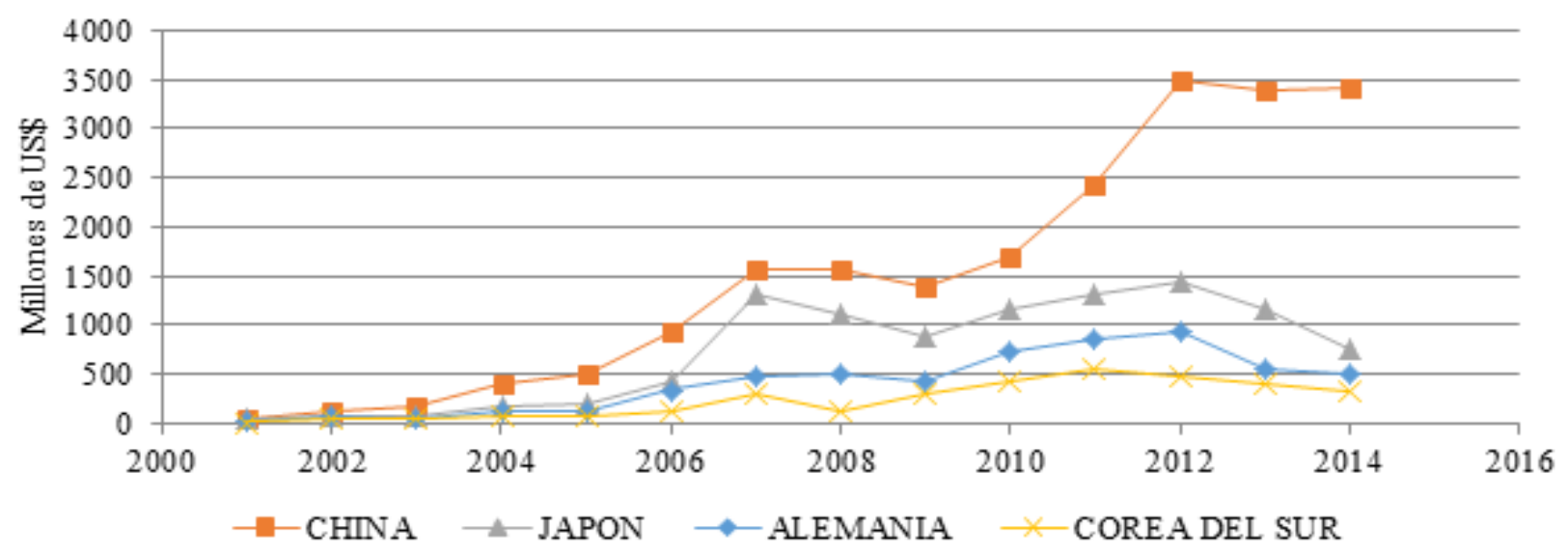

Figura 13. Valor de las exportaciones de cobre del Perú por países de destino, período 2001-2014

La demanda del cobre peruano por cada país de destino (China, Japón, Alemania y Corea del Sur) mostró una relación directa con la demanda total de cobre de cada uno de los referidos países, lo que se evidenció con el coeficiente de correlación entre las tasa de crecimiento anual del valor de las exportaciones de cobre de Perú a cada uno de los países y de la demanda total de cobre de cada uno de los referidos países (Tabla 8).

Tabla 8. Coeficiente de correlación entre la tasa de crecimiento anual del valor de las exportaciones del mineral de cobre del Perú a cada uno de los países y del valor de las importaciones totales de cobre de cada uno de los países de destino. Período 2002-2014

\begin{tabular}{ccccc}
\hline Período & China & Japón & Alemania & Corea del Sur \\
\hline $2002-2014$ & 0.643 & 0.534 & 0.456 & 0.438 \\
\hline
\end{tabular}

Cabe indicar que la participación del cobre peruano en el valor total de las importaciones de cobre de cada uno de los países de destino (China, Japón, Alemania y Corea del Sur) en el largo plazo ha mostrado una tendencia creciente, pero en el corto plazo ha sido fluctuante (Figura 14). Pero se notan caídas simultáneas de la referida participación en los cuatro países en los años 2013 y 2014.

En el caso de China, la participación del valor de las exportaciones del cobre peruano en el total de la demanda de cobre por el referido país, ha sido creciente en el largo plazo, pero fluctuante en el corto plazo, habiendo llegado a $21.3 \%$ en el 2012, pero disminuyó a $16.9 \%$ en el 2014. Comportamiento similar ocurrió con Japón, Alemania y Corea del Sur, que en el 2014 disminuyó a 7.8\%, 21\% y $9.5 \%$ respectivamente (Tabla 9). Esto presentó la posibilidad de que el Perú recupere la participación que tuvo en el 2012, en cuyo año fue mayor en los cuatro países mencionados.

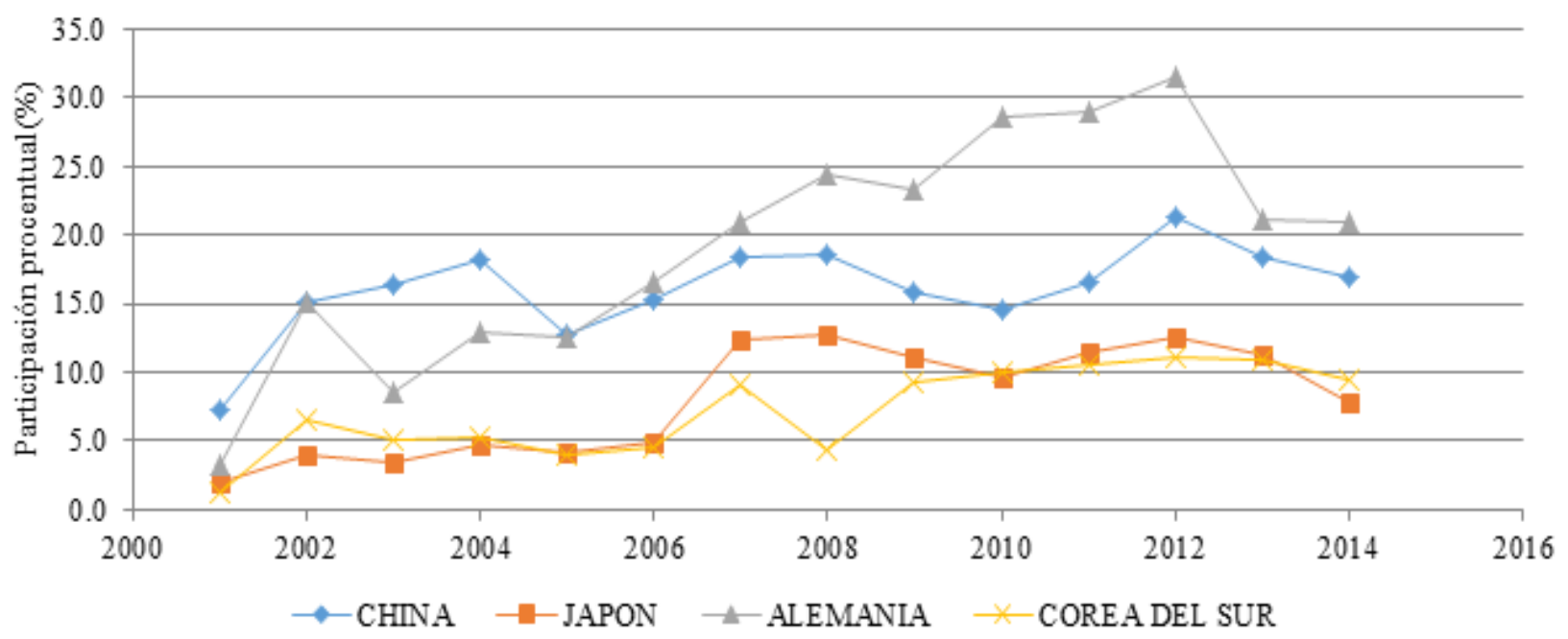

Figura 14. Participación porcentual del cobre peruano en el valor total de las importaciones de cobre de cada uno de los principales países de destino, período 2001-2014 
Tabla 9. Participación porcentual del cobre peruano en el valor total de las importaciones de cada uno de los principales países de destino, período 2001-2014

\begin{tabular}{ccccc}
\hline & China & Japón & Alemania & $\begin{array}{c}\text { Corea del } \\
\text { Sur }\end{array}$ \\
\hline 2001 & 7.2 & 1.9 & 3.3 & 1.2 \\
2002 & 15.1 & 4.0 & 15.0 & 6.5 \\
2003 & 16.4 & 3.4 & 8.5 & 5.0 \\
2004 & 18.1 & 4.8 & 12.9 & 5.3 \\
2005 & 12.7 & 4.1 & 12.6 & 3.9 \\
2006 & 15.3 & 4.9 & 16.5 & 4.5 \\
2007 & 18.3 & 12.4 & 20.9 & 9.2 \\
2008 & 18.5 & 12.7 & 24.5 & 4.4 \\
2009 & 15.9 & 11.0 & 23.3 & 9.2 \\
2010 & 14.5 & 9.6 & 28.7 & 10.0 \\
2011 & 16.6 & 11.5 & 29.0 & 10.5 \\
2012 & 21.3 & 12.6 & 31.4 & 11.1 \\
2013 & 18.4 & 11.4 & 21.1 & 10.8 \\
2014 & 16.9 & 7.8 & 21.0 & 9.5 \\
\hline
\end{tabular}

El valor de las importaciones totales de mineral de cobre (proveniente del todo el mundo) por los principales países de destino (China, Japón, Alemania y Corea del Sur) han mostrado una tendencia creciente en el largo plazo. Pero con excepción de China, los otros países cayeron en los años 2012, 2013 y 2014 (Figura 15). Pero de mantenerse la tendencia creciente para el caso de China, y de recuperarse en los casos de Japón, Alemania y Corea del Sur, se presenta una excelente oportunidad para el Perú en revertir la tendencia decreciente del valor de sus exportaciones de cobre observado en los años $2013(-8.5 \%)$ y $2014(-9.6 \%)$, y del estancamiento del volumen de sus exportaciones de cobre: 2013(-0.1\%), 2014(-0.1\%).

\section{Relación entre el PBI mundial y el volumen de importación mundial de cobre}

En el período 2001-2014, se observa una relación directa entre la variación porcentual del PBI mundial y la variación porcentual del volumen de importación mundial de cobre (Figura 16). Con excepción de los años 2009, 2010 y 2011, el crecimiento del PBI mundial explicaría el crecimiento del volumen de importación mundial de cobre, esto debido a los usos diversos que se da al cobre en sectores importantes de las economías de los países (inmobiliario, industria, energía, comunicaciones y otros). Cabe notar que en el 2009, a pesar que el PIB mundial cayó en $-1.7 \%$, el volumen de importación mundial de cobre aumentó en $11 \%$, este incremento significativo se explicaría por la caída del precio internacional del cobre, que en el referido año fue de $-18.6 \%$. Si bien es cierto que el PBI mundial creció en $4.33 \%$ y $3.12 \%$ en los años 2010 y 2011 , el volumen de importación mundial de cobre se redujo en $-2.6 \%$ y $-4 \%$ respectivamente, esto sería consecuencia del incremento significativo del stock mundial de cobre en los países importadores dado en el año 2009 (11\%), y al incremento de los precios del cobre en $48.4 \%$ (2010) y 20.2\% (2011).

\section{Relación entre el volumen de importación mundial de cobre y el precio del cobre}

En el período 2001-2014, se observó una relación inversa entre la variación porcentual del volumen de importación mundial de cobre y la variación porcentual del precio del cobre (Figura 17), evidenciada además por un coeficiente de correlación de -0.51 . Por tanto, una caída o aumento del precio del cobre propiciaría un aumento o disminución del volumen de importación mundial de cobre. Pero De la Cuba y Ferreyra (2016) expresaron que la caída del precio del cobre radicó en un consumo mundial muy por debajo de lo esperado. Cabe indicar, que un aumento significativo

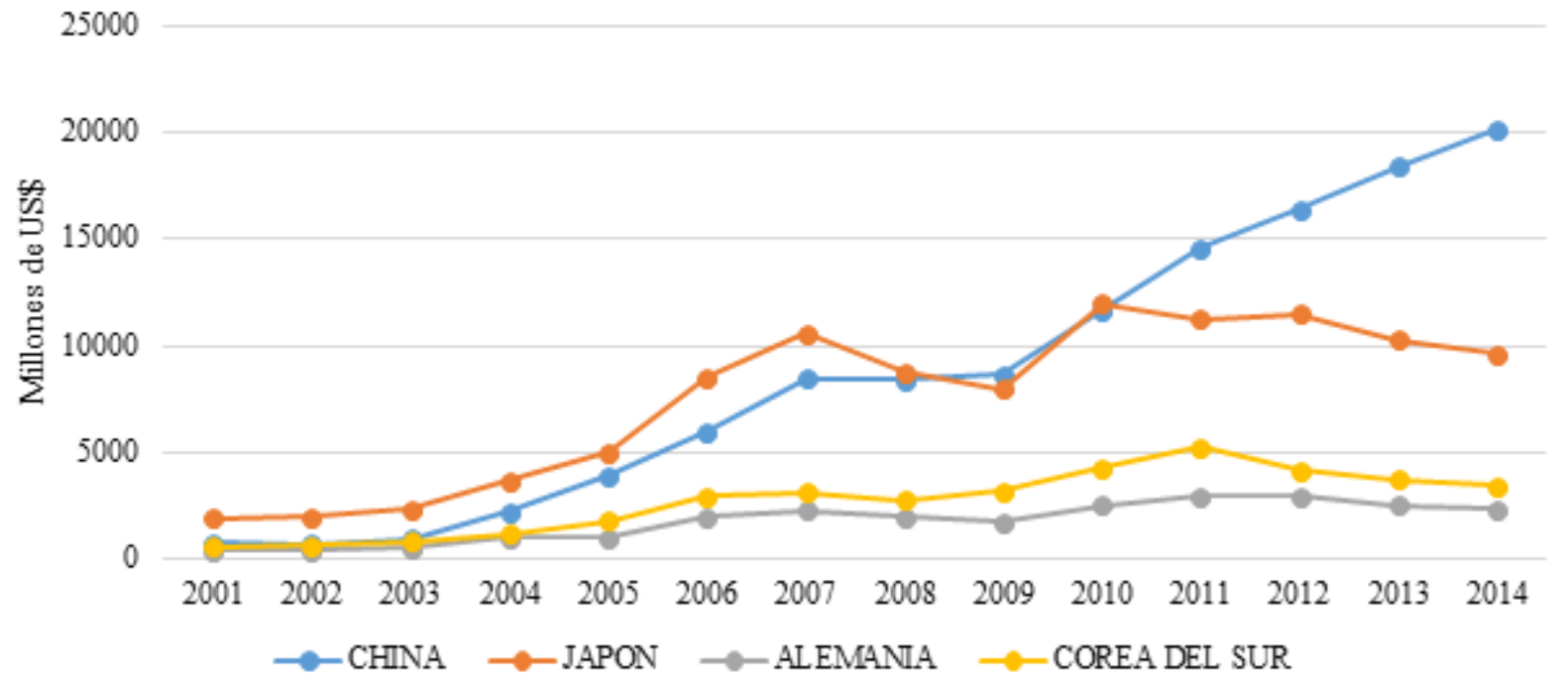

Figura 15. Comportamiento del valor de las importaciones totales de mineral de cobre por los principales países de destino, período 2001-2014 
del precio del cobre coincide con un aumento moderado del volumen de importación mundial de cobre, como lo observado en los años 2004, 2005 y 2006, en los que el precio del cobre de incrementó en $64.7 \%$, 33.8\% y $73.3 \%$, y el volumen de importación mundial de cobre creció $5.9 \%$, $6.5 \%$ y $2 \%$ respectivamente. Por lo que el crecimiento moderado del volumen de importación mundial en los referidos años se explicaría por el crecimiento significativo del PBI mundial, que fue de $4.45 \%, 3.85 \%$ y $4.34 \%$ en los años 2004, 2005 y 2006 respectivamente.
Al realizarse una regresión lineal múltiple para determinar la relación entre el volumen de importación mundial de cobre, el precio del cobre y el PIB mundial (período 2001-2014), se observó que el volumen de importación mundial motró una relación negativa con el precio del cobre (coeficiente -0.166), y una relación positiva con el PBI mundial (coeficiente 3.815), Tabla 10. Por tanto, el crecimiento del PIB mundial conllevaría a un aumento de la importación mundial de cobre, y se evidencia nuevamente la relación inversa entre el volumen de importación mundial y el precio del cobre.

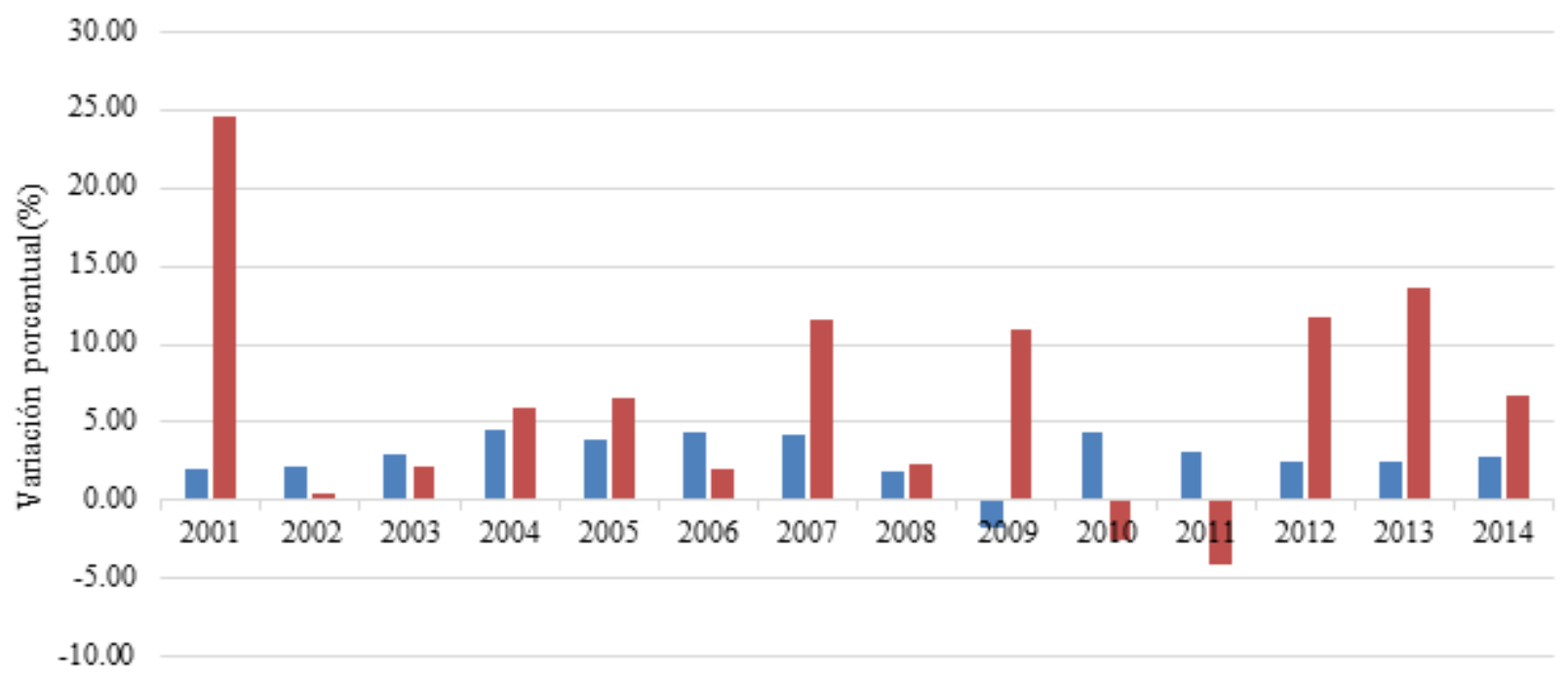

" PBI MUNDIAL "VOLUMEN DE IMPORTACION MUNDIAL DE COBRE"

Figura 16. Variación porcentual del PBI Mundial y del volumen de importación mundial de cobre, período 2001-2014

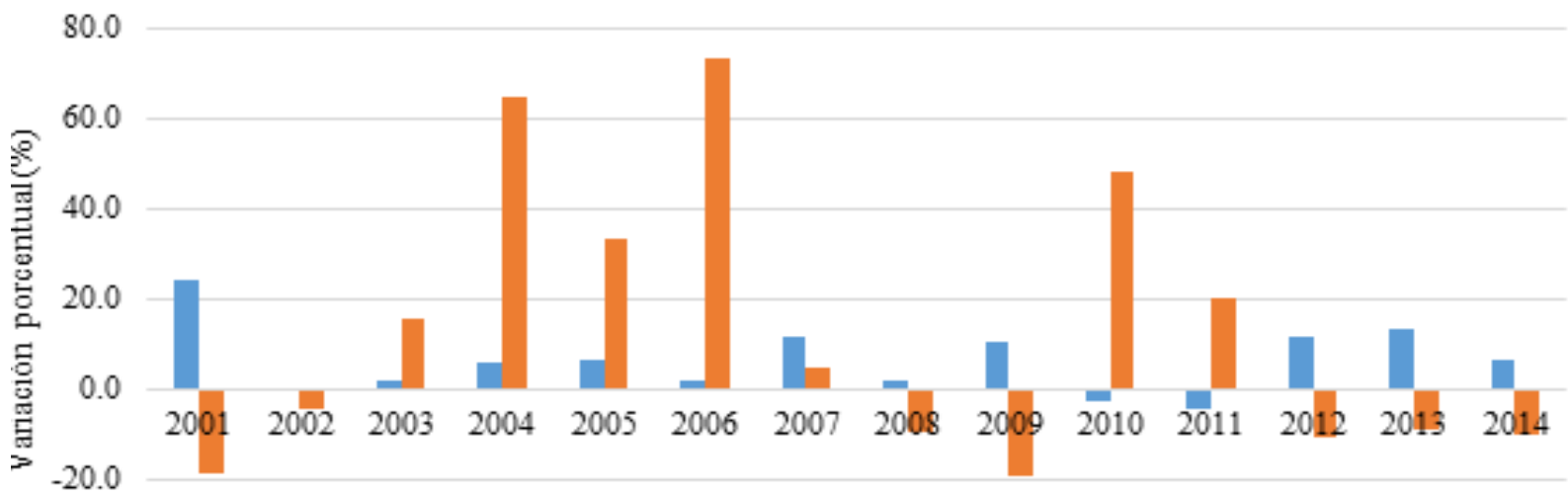

$-40.0$

-VOLUMEN DE IMPORTACION MUNDIAL DE COBRE

- PRECIO DEL COBRE

Figura 17. Variación porcentual del volumen de importación mundial de cobre y del precio del cobre, período 2001-2014 
Tabla 10. Regresión lineal múltiple entre los índices del volumen de importación mundial de cobre, del precio del cobre y del PBI mundial, período 2001-2014

\begin{tabular}{|c|c|c|c|c|}
\hline \multicolumn{5}{|c|}{ Dependent Variable: INDVOL } \\
\hline \multicolumn{5}{|c|}{ Date: $05 / 15 / 17$ Time: $18: 53$} \\
\hline \multicolumn{5}{|c|}{ Sample: 20002014} \\
\hline \multicolumn{5}{|c|}{ Included observations: 15} \\
\hline \multicolumn{5}{|c|}{$\mathrm{INDVOL}=\mathrm{C}(1)+\mathrm{C}(2) *$ INDPREC $+\mathrm{C}(3) *$ INDPBI } \\
\hline & Coefficient & Std. Error & t-Statistic & Prob. \\
\hline $\mathrm{C}(1)$ & -261.0656 & 25.79129 & -10.12224 & 0.0000 \\
\hline $\mathrm{C}(2)$ & -0.166283 & 0.028523 & -5.829781 & 0.0001 \\
\hline$C(3)$ & 3.815950 & 0.266358 & 14.32640 & 0.0000 \\
\hline R-squared & 0.978499 & Mean deper & ndent var & 162.0327 \\
\hline $\begin{array}{l}\text { Adjusted } \\
\text { R-squared }\end{array}$ & 0.974915 & S.D. depen & dent var & 38.08815 \\
\hline $\begin{array}{l}\text { S.E. of } \\
\text { regression }\end{array}$ & 6.032485 & Akaike info & o criterion & 6.609052 \\
\hline $\begin{array}{l}\text { Sum squared } \\
\text { resid }\end{array}$ & 436.6905 & Schwarz cri & citerion & 6.750662 \\
\hline Log likelihood & -46.56789 & Hannan-Qu & uinn criter. & 6.607543 \\
\hline F-statistic & 273.0521 & Durbin-Wa & atson stat & 2.112971 \\
\hline Prob(F-statistic) & 0.000000 & & & \\
\hline
\end{tabular}

\section{Conclusiones}

El valor de las exportaciones del cobre peruano fue determinado principalmente por la demanda de China, Japón, Alemania y Corea del Sur, que representaron el $72 \%$ en el 2014. En el corto plazo, el volumen y el valor de las exportaciones del cobre peruano evidenciaron un comportamiento estacional, mostrando caídas en sus tasas de crecimiento trimestral, coincidiendo con los fríos inviernos de los cuatro países mencionados anteriormente. En cuanto al precio del cobre, este muestra una relación directa con el volumen de las exportaciones del cobre peruano, evidenciado por una correlación positiva entre sus series trimestrales; con excepción de los años 2009, 2010, 2011 y 2014, en los cuales el volumen de las exportaciones del cobre peruano no creció. Por tanto, en el corto plazo, ante un incremento de la demanda mundial, aunado al incremento del precio, se estimularía el incremento del volumen y valor de las exportaciones del cobre peruano. En el período de estudio, el incremento de $800 \%$ del valor de las exportaciones de cobre del Perú (del 2001 al 2014), se explica por el incremento de 104,8\% del volumen de las exportaciones y el incremento de $340 \%$ del precio del cobre. El valor de las exportaciones del cobre peruano puede aumentar en los próximos años, al menos alcanzar nuevamente el valor que tuvo en el 2012 (10.730,94 millones US\$), y recuperar la participación porcentual en el valor de las importaciones de cobre por parte de China, Japón, Alemania y Corea del Norte que ostentaba también en el 2012. Por tanto, la demanda del cobre peruano por parte China, Japón, Alemania y Corea del Norte ha sido la variable más relevante, que junto con el comportamiento del precio internacional del cobre ha influenciado directamente en el comportamiento fluctuante en el corto plazo y la tendencia creciente en el largo plazo del valor de las exportaciones de cobre del Perú, y de manera indirecta ha influenciado el PBI real de los principales demandantes del cobre peruano.

\section{Literatura citada}

Banco Central de Reserva del Perú. Recuperado de : $\underline{\text { http:// }}$ www.bcrp.gob.pe/

Banco Central de Reserva del Perú. Memoria Anual 2006. Recuperado de: http://www.bcrp.gob.pe/publicaciones/ memoria-anual/memoria-2006.html

Banco Mundial. s.f. Recuperado de: http://datos. bancomundial.org/indicador/NY.GDP.MKTP. KD.ZG?LOCATIONS=KR

De la Cuba, M. y Ferreyra, J. 2016. Evolución y perspectiva del precio del cobre. Revista Moneda 165: 43-46.

Rojas, R. 2017. Orgullo que une y produce. Revista: De Gestión 66: 18-25.

The Observatory of Economics Complexity (OEC). REecuperado de: http://atlas.media.mit.edu/es/ visualize/tree map/hs92/export/per/show/2603/2014/ 